\title{
Sonography of Common Peripheral Nerve Disorders With Clinical Correlation
}

\author{
Jon A. Jacobson, MD, Thomas J. Wilson, MD, Lynda J.-S. Yang, MD
}

Received May 20, 2015, from the Departments of Radiology (J.A.J.) and Neurosurgery (T.J.W., L.J.-S.Y.), University of Michigan, Ann Arbor, Michigan USA. Revision requested June 11, 2015. Revised manuscript accepted for publication July 13, 2015.

Address correspondence to Jon A. Jacobson, $M D$, Department of Radiology, University of Michigan, 1500 East Medical Center Dr, 2910L, Ann Arbor, MI 48103 USA.

E-mail:jjacobsn@umich.edu
Sonography is now considered an effective method to evaluate peripheral nerves. Low cost, high resolution, the ability to image an entire limb in a short time, and dynamic assessment are several of the positive attributes of sonography. This article will review the normal appearance of peripheral nerves as shown with sonography. In addition, the most common applications for sonography of the peripheral nerves will be reviewed, which include entrapment neuropathies, intraneural ganglion cyst, nerve trauma, and peripheral nerve sheath tumors. Clinical information related to nerve disorders is also included, as it provides valuable information that can be obtained during sonographic examinations, increasing diagnostic accuracy.

Key Words - entrapment; musculoskeletal ultrasound; nerves; peripheral; sonography; trauma; tumor

S onography has been shown to be an excellent method to assess peripheral nerves, showing high accuracy in the diagnosis of many nerve disorders. With sonography, the peripheral nerves of an entire extremity can be visualized in high resolution in less time than is required with magnetic resonance imaging. With real-time examination of a peripheral nerve, direct feedback can be obtained from the patient, as transducer pressure over an abnormal nerve or neuroma commonly elicits symptoms, which serves to increase diagnostic accuracy. Evaluation of peripheral nerves dynamically either during joint movement or muscle contraction is easily completed using sonography. ${ }^{1}$ The purpose of this article is to review the normal sonographic appearance of peripheral nerves and to review common nerve disorders, including nerve entrapment, intraneural ganglion cyst, nerve trauma, and peripheral nerve sheath tumors.

\section{Sonography of Normal Peripheral Nerves}

The characteristic appearance of peripheral nerves on sonography is best appreciated in the short axis relative to the nerve, where the individual nerve fascicles will appear hypoechoic, surrounded by hyperechoic connective tissue (Figure 1). ${ }^{2}$ This appearance is likened to a honeycomb. The more proximal nerve trunks are surrounded by hyperechoic fat, and each nerve trunk can be followed distally until only one nerve fascicle remains. In the long axis relative to the nerve, the fascicular appearance can also be appreciated, with 
individual hypoechoic nerve fascicles seen surrounded by hyperechoic connective tissue. This appearance is in contrast to normal tendons, which are hyperechoic with a fibrillar or fiberlike appearance on sonography. ${ }^{2}$ When imaging peripheral nerves in the short axis, it is important that the sound beam is perpendicular to the imaged nerve so that the connective tissue layers appear hyperechoic, which makes the hypoechoic nerve fascicles more conspicuous. Angling the sound beam will cause the hyperechoic connective tissue layers to become hypoechoic due to anisotropy, making the peripheral nerve less conspicuous.

\section{Peripheral Nerve Entrapment}

The hallmark of peripheral nerve entrapment as shown with sonography is hypoechoic enlargement of the peripheral nerve at and proximal to the level of entrapment. Depending on the size of the osteofibrous tunnel or other site of entrapment, the nerve will then transition to a

Figure 1. Images from a 50-year-old man with a normal median nerve at the carpal tunnel. Sonography over the volar wrist crease shows the fascicular appearance of the normal median nerve (arrowheads) in the short axis (A) and long axis (B), with hypoechoic nerve fascicles and surrounding hyperechoic connective tissue. Findicates flexor tendons; $L$, lunate; P, palmaris longus; and $\mathrm{R}$, radius.
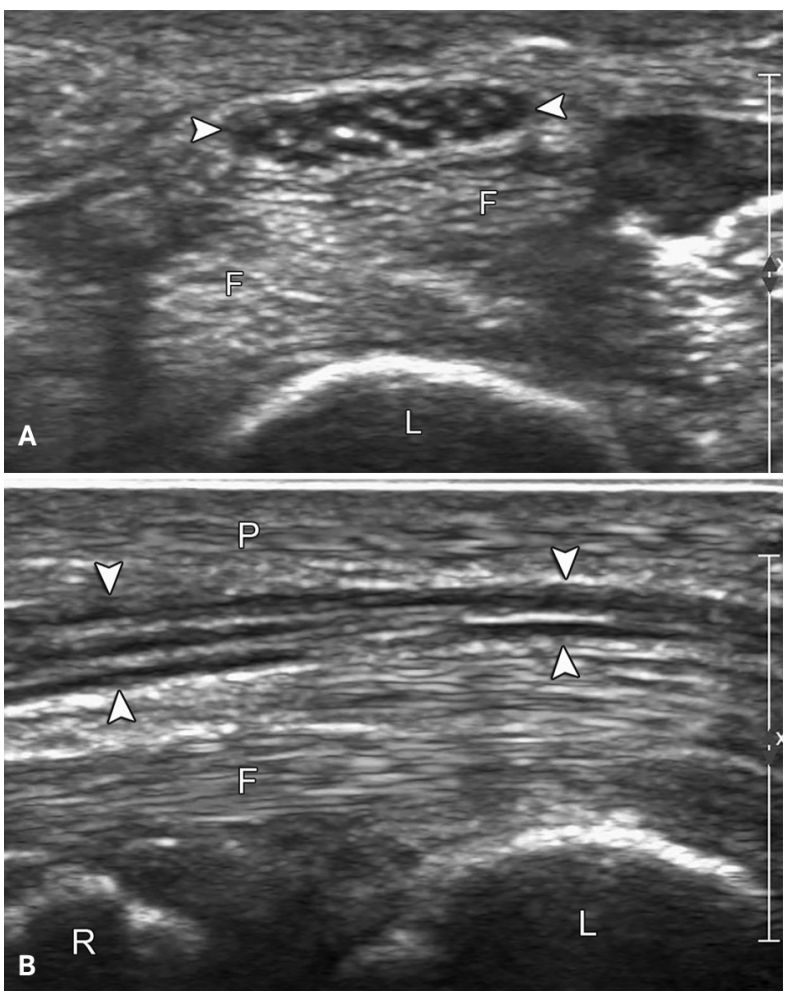

normal size as it enters into its respective canal or space. ${ }^{3}$ The earliest sonographic sign of entrapment is decreased echogenicity of the connective tissue layers of a nerve trunk, making the nerve appear globally hypoechoic. Later, the nerve may become enlarged. Transducer pressure over the abnormal nerve segment will typically elicit symptoms in the distribution of the affected nerve. The distal innervated muscle should also be assessed for denervation changes, which will appear as abnormally increased echogenicity and later atrophy as the muscle bulk decreases in size (Figure 2). When imaging for entrapment neuropathies, it is important to target the sites known to cause nerve entrapment where the affected nerve typically passes through an osteofibrous tunnel.

Figure 2. Images from a 62-year-old man with brachial plexus injury and forearm muscle atrophy. Sonography over the volar forearm (A) shows increased echogenicity and decreased size of musculature (arrows), representing atrophy. Note normal muscle echo texture and size (arrows) in the contralateral asymptomatic forearm (B). R indicates radius; and $U$, ulna.
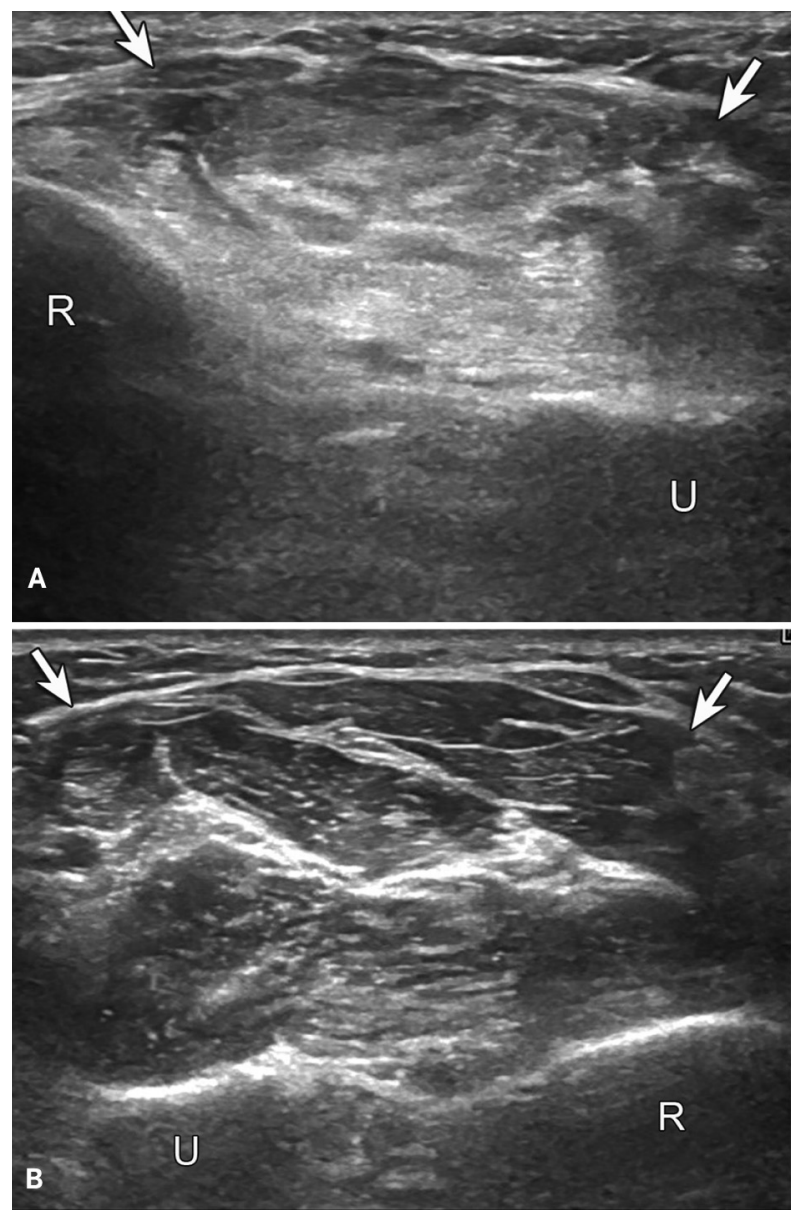
Common entrapment neuropathies include the median nerve at the carpal tunnel and ulnar nerve at the cubital tunnel. Patients with entrapment neuropathies often present with weakness of the muscles innervated by the entrapped nerve with sparing of the muscles innervated proximal to the point of entrapment. Thus, knowing the pattern of innervation can help localize the site of entrapment. In addition, patients often present with pain or paresthesias in the sensory distribution of the entrapped nerve. Provocative maneuvers that increase the pressure on the nerve such as the Phalen test for the carpal tunnel will exacerbate pain or paresthesias, similar to that elicited via direct pressure with the ultrasound transducer. Likewise, the Tinel test at the site of entrapment will also often elicit pain or paresthesias. In addition to sonography, electrodiagnostic testing can be a useful diagnostic modality in identifying entrapment neuropathies. Electrodiagnostic findings of entrapment neuropathies often include prolonged sensory and motor latencies across the point of entrapment, consistent with that seen by sonographic examination. Sonography and electrodiagnostic testing complement each other, as shown in evaluation of ulnar neuropathy at the elbow, in which the addition of sonography to electrodiagnostic tests increases sensitivity from $78 \%$ to $98 \%$ and additionally localizes lesions not identified with electrodiagnostic tests alone. ${ }^{4,5}$

\section{Carpal Tunnel Syndrome}

The carpal tunnel represents an osteofibrous canal bordered by the transverse carpal ligament or flexor retinaculum at the volar aspect and the carpal bones dorsally. The flexor retinaculum at its proximal aspect attaches to the pisiform and the scaphoid tubercle and distally from the hook of the hamate to the trapezoid tubercle. ${ }^{6}$ The median nerve and flexor digitorum superficialis and profundus tendons are located within the carpal tunnel. The adjacent flexor carpi radialis tendon is located outside the carpal tunnel, as well as the adjacent Guyon canal, containing the ulnar nerve and artery. Although median nerve entrapment may be idiopathic or from overuse, any spaceoccupying abnormality within the carpal tunnel, such as a ganglion cyst or synovitis, may cause secondary carpal tunnel syndrome.

The diagnosis of carpal tunnel syndrome is made on a clinical basis and supported by sonography, electrodiagnostic testing, or both. ${ }^{7}$ Patients typically present with numbness or paresthesias on the palmar surface of the radial three-and-a-half digits (sensory innervation of the median nerve). Patients may have reduced grip strength and, late in the course, may have atrophy of the thenar eminence.
Pain may radiate up the arm, even as far proximally as the shoulder. The pain and paresthesias often display a pattern of nocturnal worsening and exacerbation with activity, particularly activity that requires repetitive wrist flexion. On physical examination, the Phalen test and Tinel tests at the wrist are provocative maneuvers that may exacerbate pain or paresthesias. Median innervated muscles, including the first and second lumbricals, opponens pollicis, abductor pollicis brevis, and flexor pollicis brevis, may be weak on examination.

On sonography, carpal tunnel syndrome is characterized by an abnormal hypoechoic and enlarged median nerve (Figure 3). ${ }^{6,8}$ This enlargement typically occurs at the proximal edge of the flexor retinaculum but may extend proximally or less commonly distally into the carpal tunnel. The early finding of carpal tunnel syndrome, which is decreased echogenicity of the connective tissue layers around the nerve fascicles, can be subjective. Enlargement of the median nerve can be measured and is a more objective sonographic finding.

Figure 3. Images from a 68-year-old man with carpal tunnel syndrome. Sonography over the volar wrist crease short axis $(\mathbf{A})$ and long axis (B) to the median nerve shows hypoechoic enlargement of the median nerve (arrows) proximal to the carpal tunnel, with a transition (curved arrow) to normal size within the carpal tunnel (arrowheads).
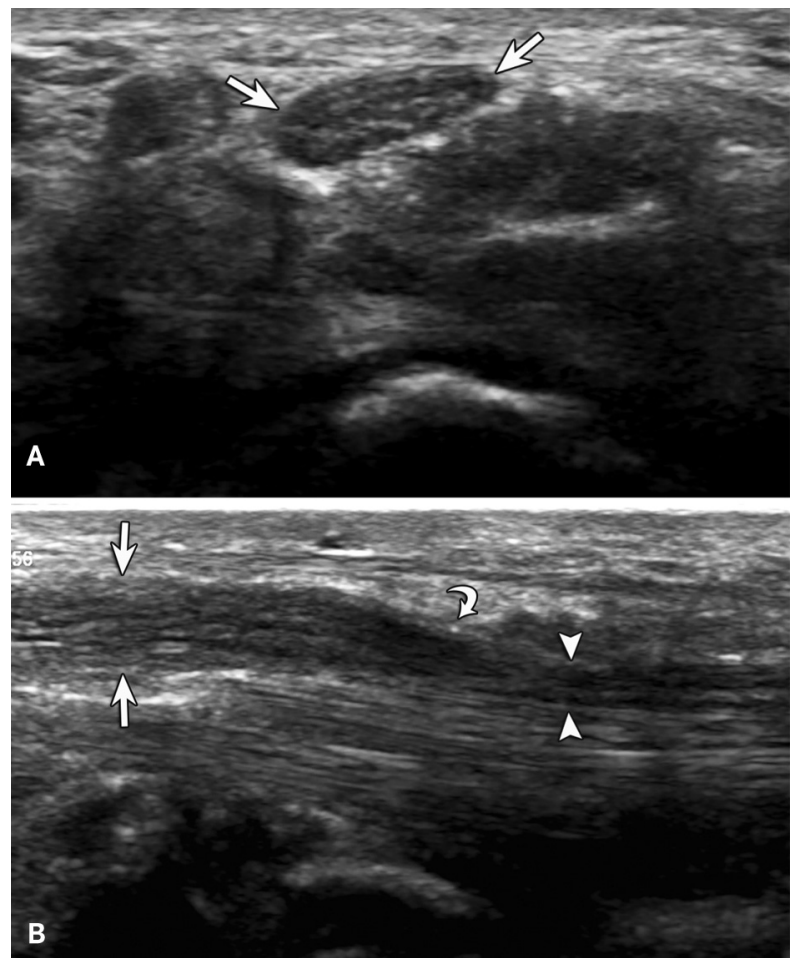
The threshold to diagnose abnormal median nerve enlargement in the setting of carpal tunnel syndrome depends on where one chooses sensitivity and specificity. In general, a normal median nerve measures less than 8 $\mathrm{mm}^{2}$ at the level of the volar wrist crease; 8 to $12 \mathrm{~mm}^{2}$ is considered borderline; and greater than $12 \mathrm{~mm}^{2}$ is considered abnormal. ${ }^{8}$ The degree of median nerve enlargement correlates with the severity of carpal tunnel syndrome as diagnosed by nerve conduction studies, with a threshold of $12.8 \mathrm{~mm}^{2}$ considered moderate and $14 \mathrm{~mm}^{2}$ considered severe carpal tunnel syndrome. ${ }^{9}$ Given that patients of different sizes may also have variable sizes of their median nerves, a change in median nerve size may be more accurate in assessment for carpal tunnel syndrome.

A study by Klauser et $\mathrm{al}^{10}$ has shown that an area difference of $2 \mathrm{~mm}^{2}$ or greater measured proximally at the level of the proximal pronator quadratus compared with maximal enlargement in the region of the wrist crease results in $99 \%$ sensitivity and $100 \%$ specificity in the diagnosis of carpal tunnel syndrome using electrodiagnostic tests as the standard of reference. In the long axis, a change in caliber of the median nerve area from proximal to distal as the median nerve enters the carpal tunnel has been termed the "notch sign." In addition, the presence of flow on color or power Doppler imaging within the median nerve can indicate carpal tunnel syndrome with $95 \%$ accuracy. ${ }^{11}$

As a normal variant, there may be a high bifurcation of the median nerve proximal to the wrist or a bifid median nerve, typically with an interposed median artery, which is present in $2.8 \%$ of the population. ${ }^{12}$ In this setting, carpal tunnel syndrome can be diagnosed if there is an increase in the combined areas of the two nerve trunks equal or greater to $4 \mathrm{~mm}^{2}$ measured from the level of the pronator quadratus to the carpal tunnel (Figure 4) ${ }^{13}$ After carpal tunnel release, the flexor retinaculum is typically thickened and displaced in a palmar direction. ${ }^{14}$ Although a normal sonographic appearance of the median nerve after carpal tunnel release may occur, median nerve size does not correlate with surgical success (Figure 5). ${ }^{15}$

\section{Cubital Tunnel Syndrome}

The cubital tunnel represents an osteofibrous tunnel containing the ulnar nerve at the elbow bordered by the two heads of the flexor carpi ulnaris, the arcuate ligament, and the ulna. ${ }^{16}$ Proximal to the cubital tunnel, the ulnar nerve lies posterior to the medial epicondyle of the humerus, superficial to the posterior band of the ulnar collateral ligament and beneath the fascia of Osborn (or cubital tunnel retinaculum), which is continuous with the arcuate ligament. ${ }^{16}$ Typical points of compression of the ulnar nerve around the elbow include the arcade of Struthers, median intermuscular septum, postcondylar groove, arcuate ligament, Osborne fascia, and potentially, when present, an accessory anconeus epitrochlearis muscle.

Patients with ulnar neuropathy typically present with pain or paresthesias in the ulnar one-and-a-half digits. They often display a temporal pattern similar to carpal tunnel syndrome with worsening of symptoms at night. Patients with ulnar neuropathy should have sensory symptoms limited to the hand, as the ulnar sensory field extends only a few centimeters proximal to the wrist crease. Altered sensation proximal to this location should prompt a search for an alternative diagnosis. Weakness of the intrinsic hand musculature, particularly the third and fourth lumbricals and both the palmar and dorsal interossei, is associated with ulnar neuropathy. Characteristic signs of ulnar neuropathy include the Froment sign (weakness of the adductor pollicis compensated by the median nerve-innervated flexor pollicis longus) and clawing of the hand. The Tinel test at the elbow may exacerbate pain or paresthesias. Sparing of the dorsal ulnar distribution should raise suspicion of

Figure 4. Images from a 79-year-old woman with a bifid median nerve and carpal tunnel syndrome. Sonography over the volar wrist crease short axis (A) and long axis (B) to the median nerve shows hypoechoic enlargement of the median nerve trunks (arrows) proximal to the carpal tunnel, with a transition to normal size within the carpal tunnel (arrowheads).

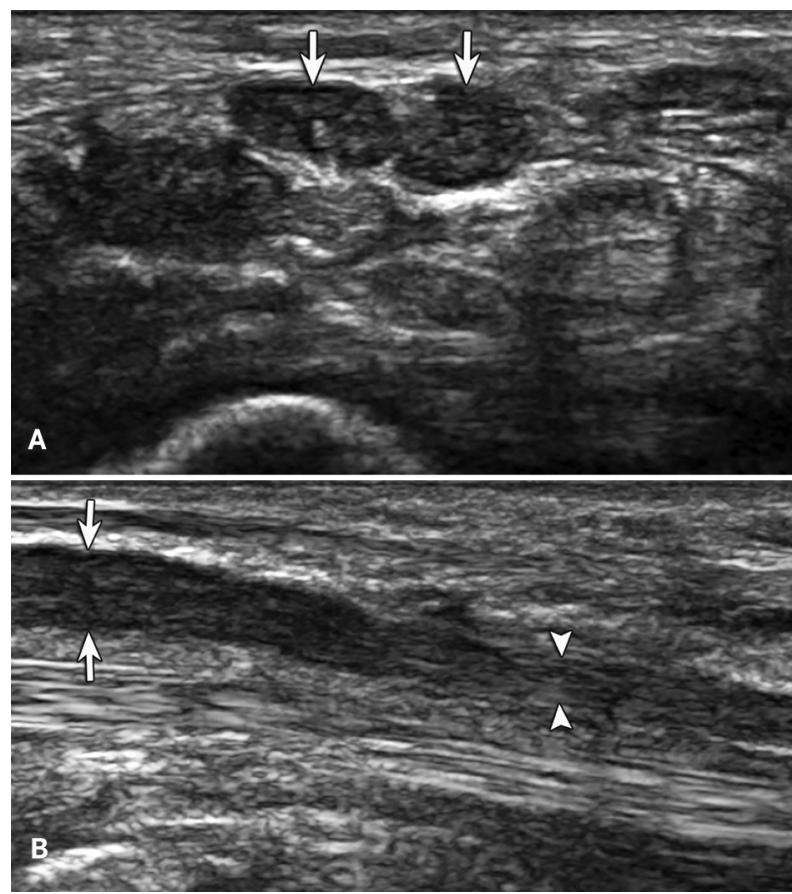


compression in the Guyon canal, rather than at the elbow, as the dorsal ulnar cutaneous branch arises proximal to the Guyon canal and thus is spared.

On sonography, cubital tunnel syndrome is characterized by hypoechoic enlargement of the ulnar nerve just proximal to the cubital tunnel at the level of the medial epicondyle (Figure 6). ${ }^{3}$ The enlarged nerve will then transition to normal size as it enters the cubital tunnel beneath the arcuate ligament, seen both short axis and long axis to the nerve. An area threshold for the diagnosis of cubital tunnel syndrome has been defined as between 7.5 and 9 $\mathrm{mm}^{2}$ or an increase in area by 1.5 times from the mid humerus to the medial epicondyle ${ }^{3}$; however, other studies have defined the upper limit of the normal ulnar area as 8 to $10 \mathrm{~mm}^{2}{ }^{217}$ This variability emphasizes the importance of real-time assessment of the ulnar nerve for enlargement, decreased echogenicity, and associated clinical symptoms. Of note, the ulnar nerve often appears hypoechoic and minimally enlarged up to $6.8 \mathrm{~mm}^{2}$ in asymptomatic individuals, which should not be misinterpreted as cubital tun-

Figure 5. Images from a 61-year-old woman with failed carpal tunnel release. Sonography over the volar wrist crease short axis $(\mathbf{A})$ and long axis (B) to the median nerve shows hypoechoic enlargement of the median nerve (arrows) with thickening of the overlying flexor retinaculum (curved arrows).
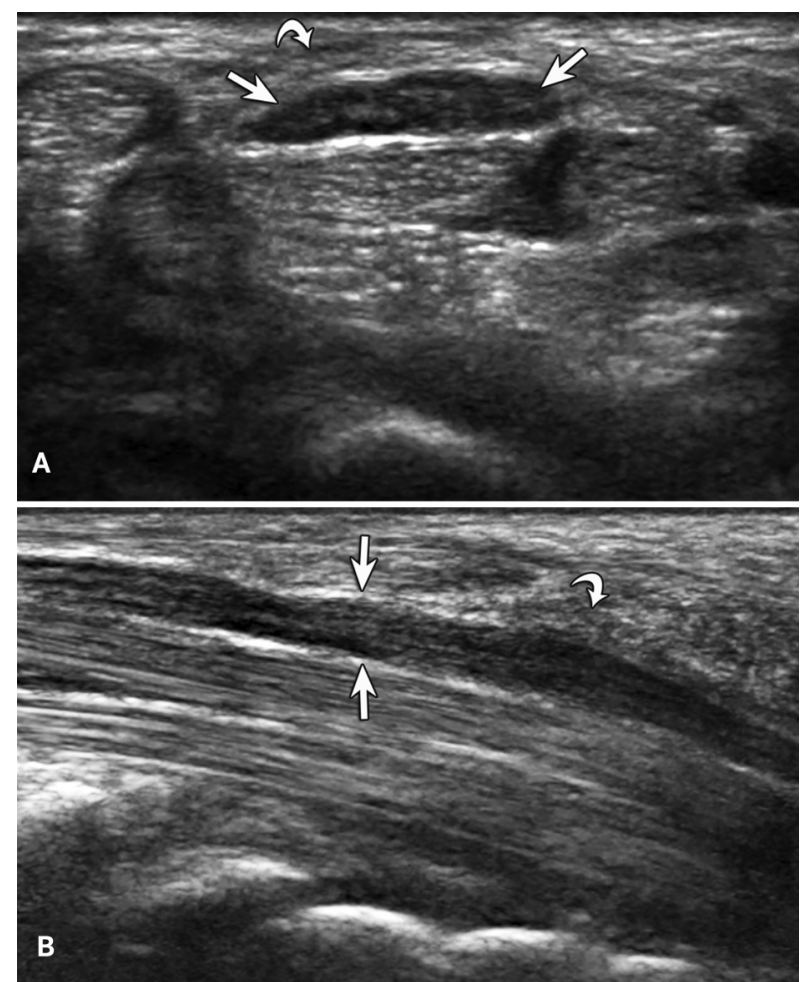

nel syndrome. ${ }^{3}$ Uncommonly, a ganglion cyst or adjacent joint process, such as synovitis, may be a secondary cause of ulnar nerve compression. The presence of an anconeus epitrochlearis, seen in up to $34 \%$ of the population, may also cause nerve compression, which appears as hypoechoic muscle superficial to the ulnar nerve in place of the fascia of Osborn (Figure 7). ${ }^{3}$ Evaluation for an anconeus epitrochlearis is best performed with elbow extension. With elbow flexion, the anconeus epitrochlearis blends in with the triceps brachii and may not be visualized.

The ulnar nerve should be assessed dynamically for abnormal subluxation and dislocation. Normally during elbow flexion, the ulnar nerve should remain posterior to the medial epicondyle. Movement of the ulnar nerve superficial and anterior to the medial epicondyle apex with elbow flexion should be considered abnormal, although potentially an asymptomatic finding in up to $20 \%$ of individuals (Figure 8). ${ }^{3}$ Transducer pressure during this dynamic assessment should be reduced so as not to inhibit

Figure 6. Images from a 52-year-old woman with cubital tunnel syndrome. Sonography over the cubital tunnel region short axis $(\mathbf{A})$ and long axis (B) to the ulnar nerve shows marked hypoechoic enlargement of the ulnar nerve (arrows) with a transition to normal size as it enters the cubital tunnel distally beneath the arcuate ligament (curved arrow). Note the fascia of Osborn (arrowhead in A). E indicates medial epicondyle of the humerus; and $\mathrm{O}$, olecranon process of the ulna.
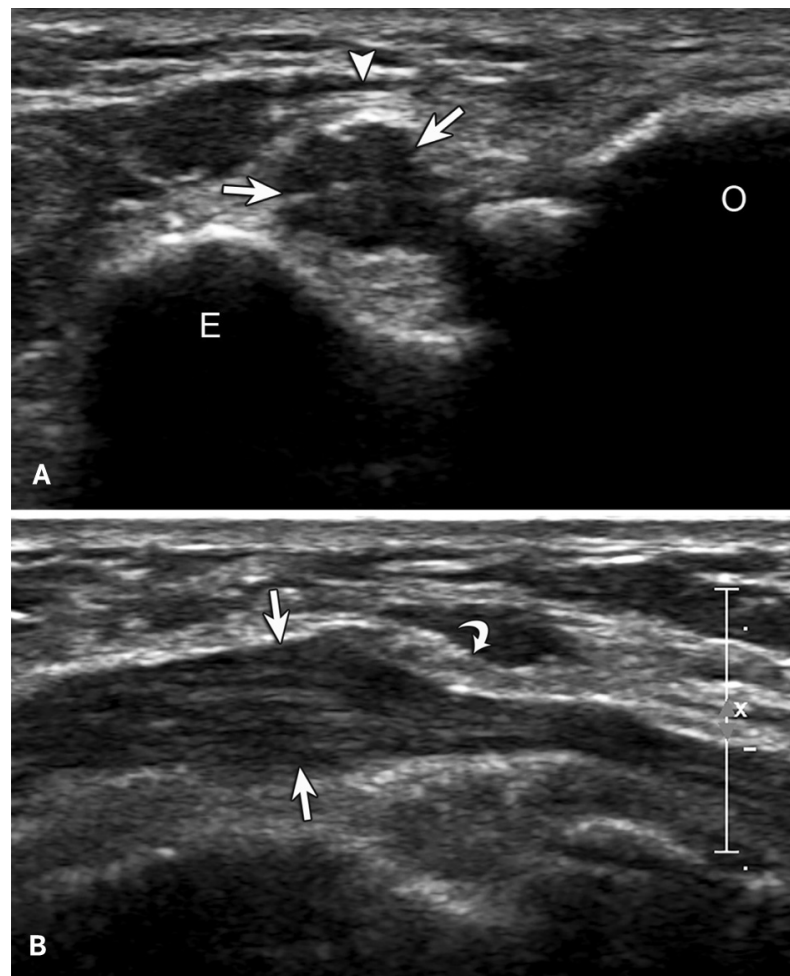
movement of the ulnar nerve. Abnormal displacement of the medial head of the triceps brachii, if present, may result in two palpable snaps, termed snapping triceps syndrome (Figure 9). ${ }^{18}$ After surgical treatment for ulnar nerve dislocation, the ulnar nerve may be found in a subcutaneous or submuscular position anterior to the medial epicondyle.

\section{Morton Neuroma}

A Morton neuroma represents an enlarged plantar digital nerve at the level of the metatarsal heads from edema and fibrosis as the result of chronic nerve entrapment under the intermetatarsal ligament. ${ }^{19}$ The most common sites for a Morton neuroma are the second and third metatarsal interspaces. ${ }^{19}$ Enlargement of the intermetatarsal bursa may be associated with a Morton neuroma.

Patients most commonly present with pain, typically burning in character, in the third web space. Pain is typi-

Figure 7. Images from a 78-year-old man with an anconeus epitrochlearis. Sonography over the cubital tunnel region short axis $(\mathbf{A})$ and long axis (B) to the ulnar nerve shows an anconeus epitrochlearis muscle (arrows) overlying the hypoechoic and enlarged ulnar nerve (arrowheads). E indicates medial epicondyle of the humerus; and O, olecranon process of the ulna.
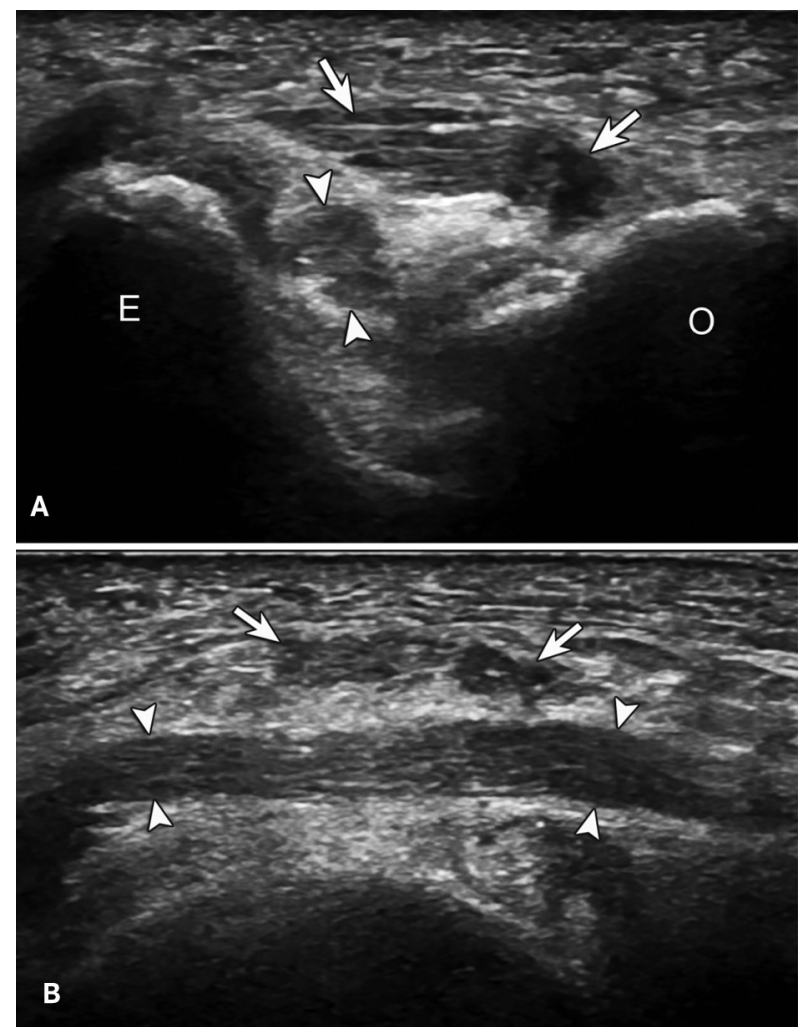

cally focal but can radiate into the toes and is often exacerbated by footwear that has a narrow toe box or by high heels. ${ }^{20}$ On clinical examination, pain can be provoked by palpation of the affected web space or by squeezing the metatarsals. The Tinel test over the affected web space can also precipitate pain. Cloke and Greiss ${ }^{21}$ have described the digital nerve stress test, which they found to have $100 \%$ sensitivity and a $95 \%$ positive predictive value. In comparison, the Mulder maneuver in their hands had $95 \%$ sensitivity and $100 \%$ specificity.

On sonography, a Morton neuroma will appear as a hypoechoic round or oval mass between the metatarsal heads, which presents as the enlarged digital nerve (Figure 10). ${ }^{22}$ Sonographic evaluation for a Morton neuroma can be performed plantar with the examiner's finger applying pressure from the dorsal aspect, or the other way around. Regardless, the Morton neuroma should be confirmed in both short and long axes relative to the digital nerve. Dynamic assessment using the Mulder maneuver is helpful to increase diagnostic accuracy. ${ }^{23}$ With the

Figure 8. Images from a 65-year-old man with ulnar nerve dislocation. Sonography over the cubital tunnel region short axis to the ulnar nerve in elbow extension (A) shows an enlarged and hypoechoic ulnar nerve (arrow). In elbow flexion (B), there is abnormal dislocation of the ulnar nerve (arrow) anterior to the medial epicondyle of the humerus (E). $O$ indicates olecranon process of the ulna; and T, triceps brachii muscle.
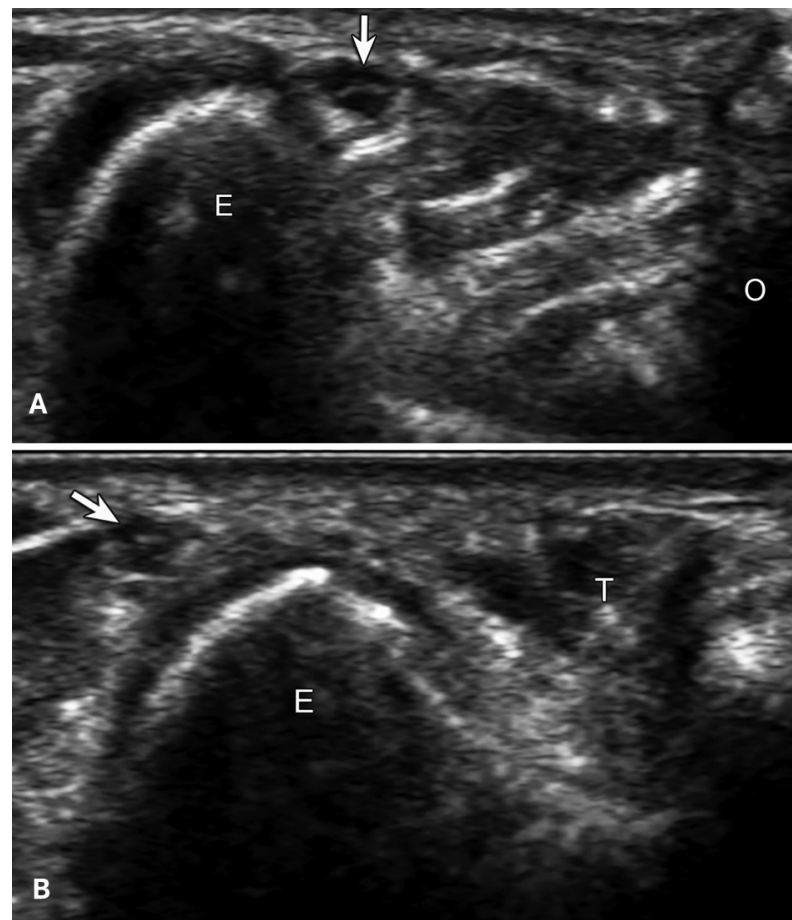
transducer placed short axis to the nerve at the plantar aspect of the foot at the level of the metatarsal heads, the metatarsals are squeezed together. During this dynamic maneuver, a Morton neuroma will snap abruptly in a plantar direction as the metatarsals become approximated and will be noncompressible. In contrast, an intermetatarsal bursa will tend to displace less and will be compressible. Of note, the detection rate for sonography in the diagnosis of Morton neuroma has been described as $79 \%$ and equal to magnetic resonance imaging, ${ }^{24}$ although another study described $100 \%$ sensitivity. ${ }^{22}$

\section{Intraneural Ganglion Cyst}

One cause of foot drop and common peroneal nerve symptoms is the presence of an intraneural ganglion cyst. The pathophysiologic mechanism of this entity has been described by Spinner et $\mathrm{al}^{25-29}$ as related to an articular branch of the common peroneal nerve that innervates the

Figure 9. Images from a 47-year-old man with snapping triceps syndrome. Sonography over the cubital tunnel region short axis to the ulnar nerve in elbow extension (A) shows the ulnar nerve (arrow). In elbow flexion (B), there is abnormal dislocation of the ulnar nerve (arrow) anterior to the medial epicondyle of the humerus $(E)$ with subluxation of the medial head (arrowheads) of the triceps brachii muscle ( $T$ ). O indicates olecranon process of the ulna.
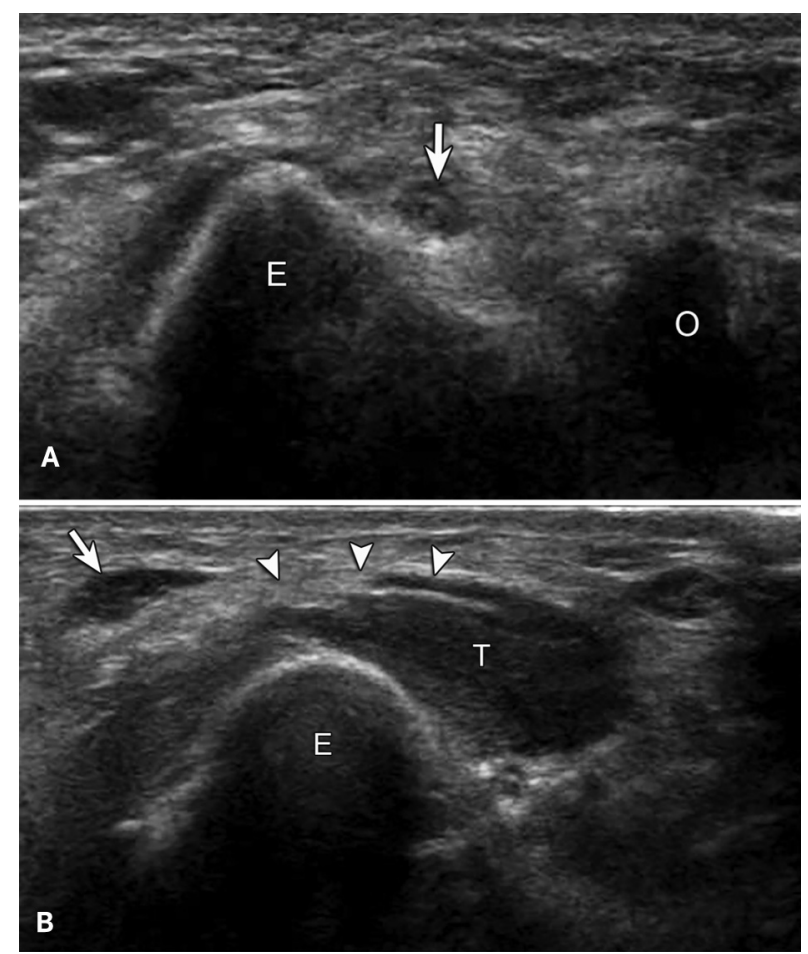

proximal tibiofibular joint. The knee joint may communicate with the proximal tibiofibular joint, and joint fluid under pressure may therefore extend into the tibiofibular joint and subsequently into the common peroneal nerve

Figure 10. Images from a 37-year-old woman with a Morton neuroma. Sonography over the plantar foot at the level of the metatarsals long axis (A) and short axis (B) to plantar digital nerves show a hypoechoic Morton neuroma (arrows) between the metatarsal heads (M). Note the digital plantar nerve (arrowheads) entering into the Morton neuroma in $\mathbf{A}$. When the metatarsals are squeezed together (Mulder maneuver), the Morton neuroma (C, arrows) displaces in a plantar direction, producing a palpable click.
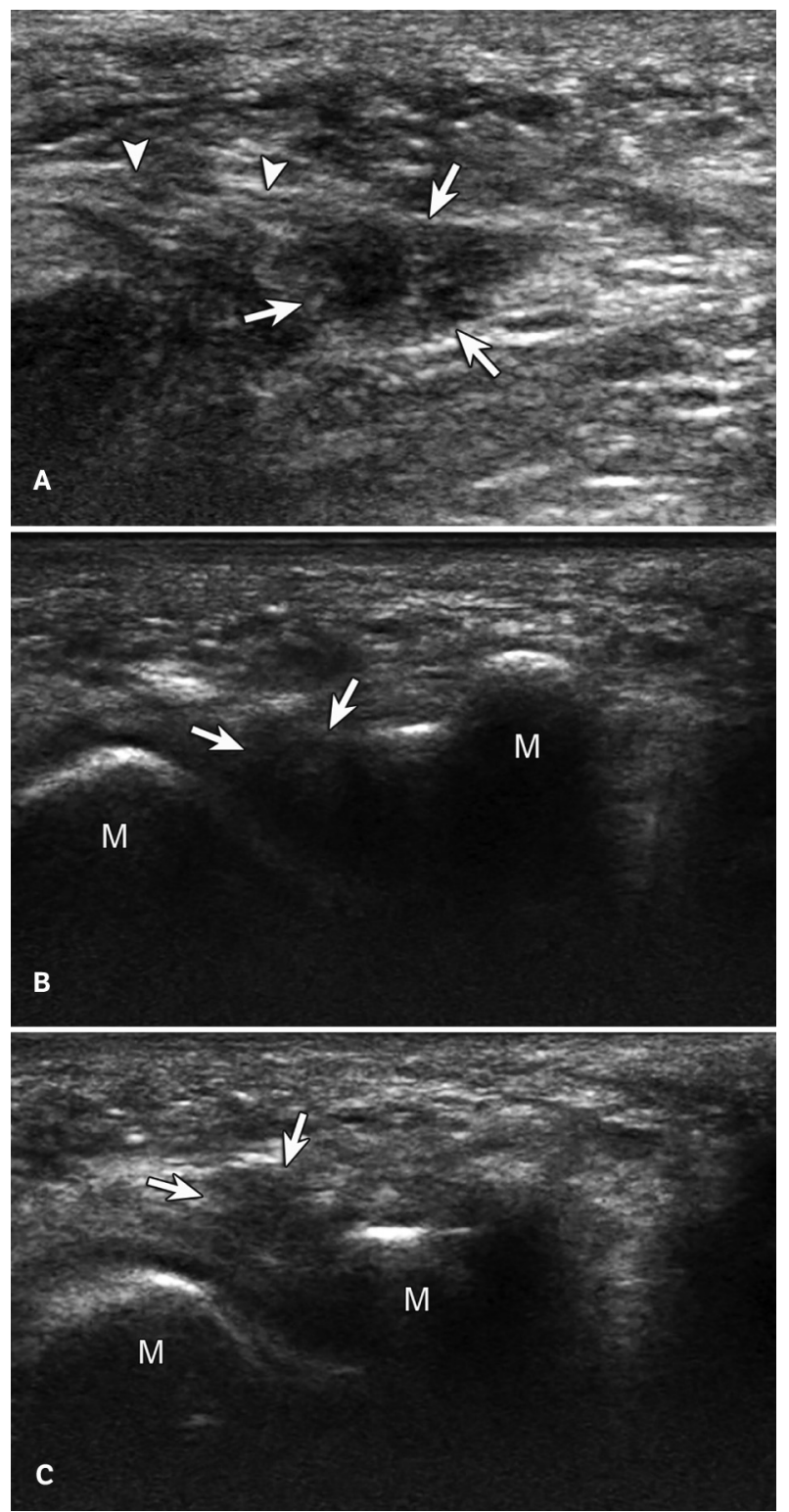
via the articular branch. The resulting intraneural ganglion cyst can extend proximally, even into the sciatic or tibial nerves. A variation of this cyst is extension directly into the tibial nerve via a different articular branch.

Patients may present with a palpable mass on the anterior and lateral aspect of the knee. However, patients may not have a palpable mass and may rather present only with neurologic symptoms of compression of the common peroneal nerve. Symptoms may include weakness of dorsiflexion and eversion, and there may be pain or paresthesias on the anterolateral lower leg and dorsum of the foot. An intraneural ganglion cyst versus other peroneal entrapment is suggested clinically by the presence of a palpable mass or by a predominance of symptoms referable to the deep peroneal nerve with dorsiflexion weakness out of proportion to eversion weakness and minimal sensory symptoms. ${ }^{30}$

On sonography, an intraneural ganglion cyst will appear as an anechoic, often multilocular fluid collection adjacent to and along the course of the common peroneal nerve (Figure 11). ${ }^{31-33}$ The smaller articular branch component is often more difficult to visualize, given its small size and nonlinear course. In addition, a ganglion cyst may be heterogeneous and more hypoechoic. The course of the ganglion cyst along the common peroneal nerve differentiates this entity from a tibiofibular joint cyst.

Figure 11. Image from a 59-year-old woman with an intraneural ganglion cyst of the common peroneal nerve. Sonography over the lateral knee proximal to the fibula shows a hypoechoic and multilocular ganglion cyst (arrow) adjacent to a compressed common peroneal nerve (arrowheads). Note increased through-transmission (curved arrow) deep to the ganglion cyst.

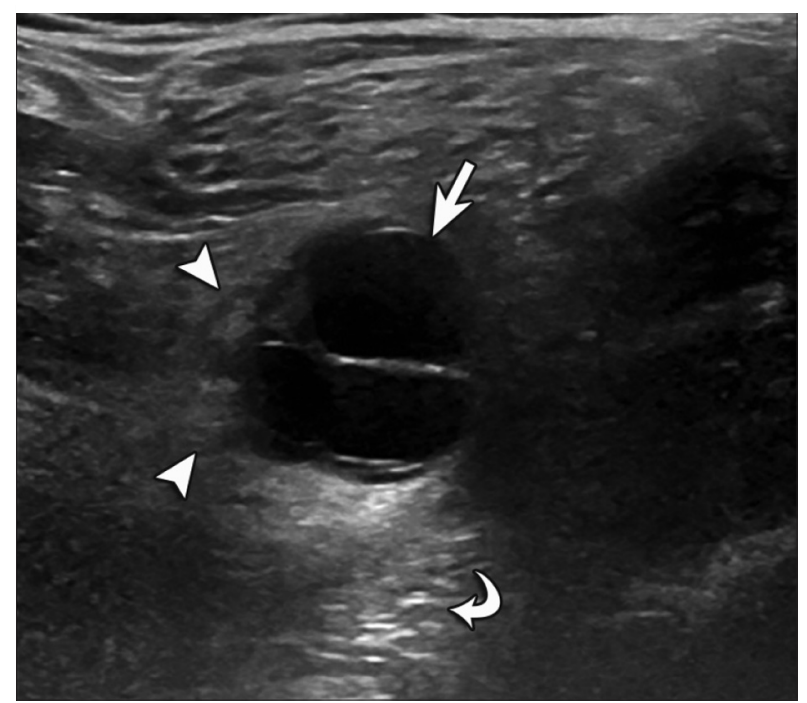

\section{Nerve Trauma}

Nerve trauma may be due to compression, traction, as well as laceration or surgical transection. ${ }^{34}$ In the latter scenario, a neuroma is an expected finding as the peripheral nerve attempts to regenerate. A delayed neurologic deficit may also be seen in the setting of trauma due to compression from an adjacent hematoma.

Compression of a peripheral nerve may cause edema, demyelination, and axonal damage. ${ }^{35}$ The affected nerve segment will appear abnormally hypoechoic and enlarged on sonography (Figure 12). Similarly, a traction injury to a nerve may cause the nerve to appear hypoechoic and enlarged (Figure 13). ${ }^{34}$ After peripheral nerve laceration or transection, a traumatic neuroma will appear as a hypoechoic enlargement of variable size. ${ }^{34,36}$ Continuity between the hypoechoic neuroma and the affected peripheral nerve is essential for an accurate diagnosis (Figure 14). Ultrasound

Figure 12. Images from a 23-year-old woman with compression injury to the radial nerve (Saturday night palsy). Sonography over the posterior humeral shaft $(\mathrm{H})$ short axis $(\mathbf{A})$ and long axis $(\mathbf{B})$ to the radial nerve shows diffuse hypoechoic enlargement of the radial nerve (arrowheads).

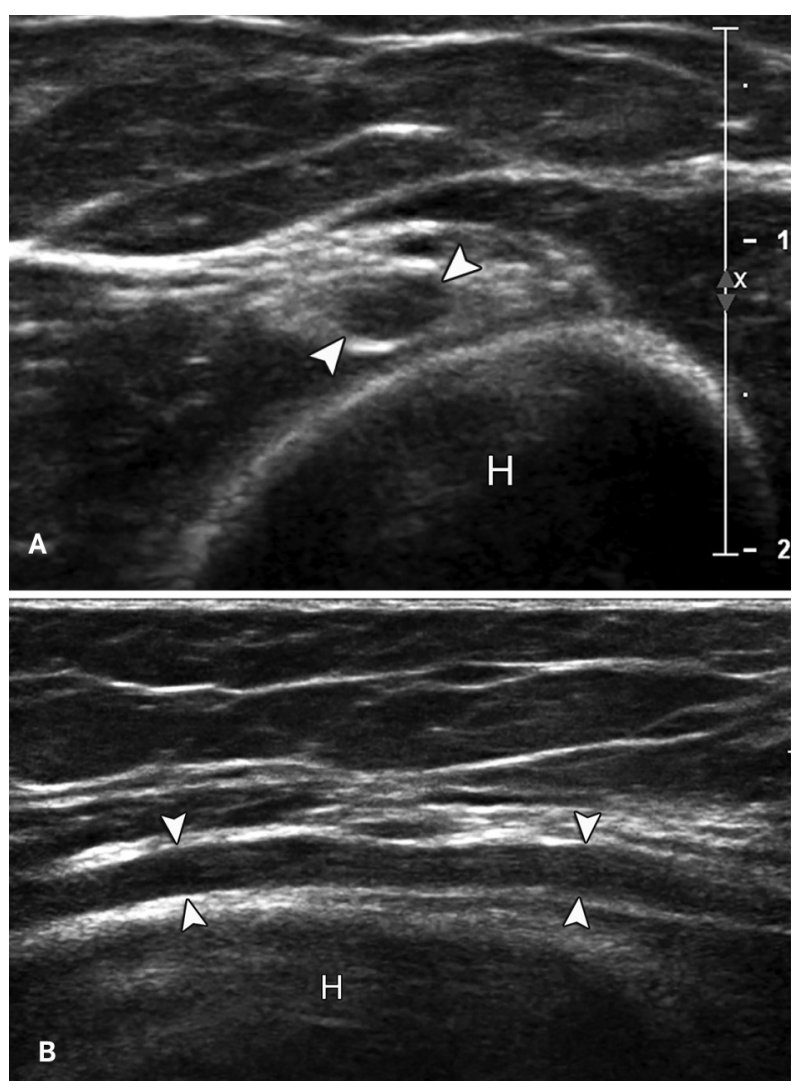


transducer pressure over the neuroma will often reproduce patient symptoms, such as phantom symptoms in the setting of extremity amputation. With complete nerve transection, retraction occurs between the two severed nerve endings. ${ }^{34,37}$ Sonography may also be used to identify a hematoma causing secondary nerve compression, which commonly appears hypoechoic and heterogeneous.

\section{Peripheral Nerve Sheath Tumors}

Benign peripheral nerve sheath tumors can be categorized as schwannomas (or neurilemomas) and neurofibromas. ${ }^{38}$ At histologic analysis, in contrast to neurofibromas, schwannomas are characterized by the presence of Antoni $A$ and Antoni B cells and are also strongly S-100 positive at immunohistochemical analysis. ${ }^{38}$ Schwannomas may be solitary or uncommonly multiple (termed schwannomatosis). Neurofibromas may also be solitary or multiple, with the latter seen in neurofibromatosis. Other types of involvement with neurofibromatosis include plexiform and diffuse neurofibromas, with the latter most commonly located in the subcutaneous region of the head and neck. ${ }^{38}$

Patients with benign peripheral nerve sheath tumors can present in a variety of ways but most commonly present with a painless, palpable lump. Symptoms, however, may include pain and paresthesias in the distribution of the affected nerve (particularly with direct pressure on the mass) or, less commonly, weakness of the muscles innervated by the affected nerve and localized pain at the site of the mass. Severe pain, early motor deficits, or rapid growth should increase suspicion for malignancy, particularly in

Figure 13. Image from a 38-year-old woman with traction injury and a neuroma of the spinal accessory nerve branch proven at surgery. Sonography short axis to the sternocleidomastoid muscle (SM) shows hypoechoic enlargement (arrows) of a spinal accessory nerve branch (arrowheads), representing a neuroma.

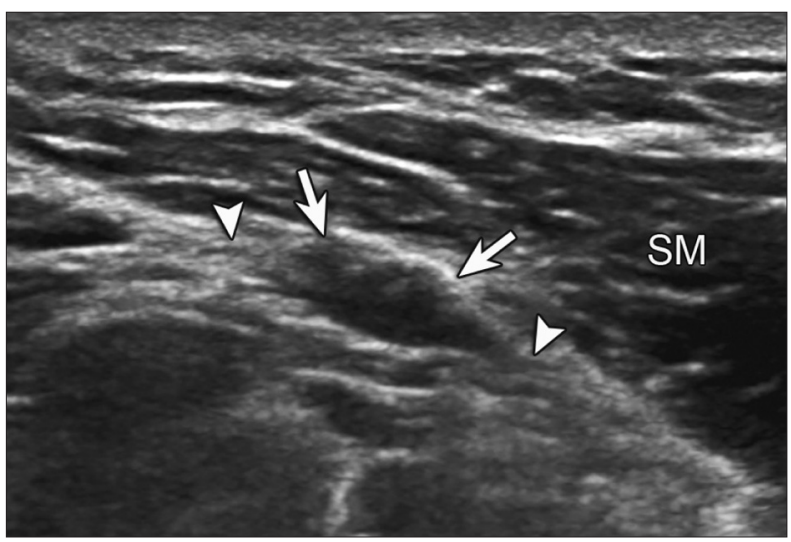

the context of neurocutaneous syndromes, such as neurofibromatosis types 1 and 2 .

On sonography, both a schwannoma (Figure 15) and a neurofibroma (Figure 16) have the common and characteristic appearance of a hypoechoic mass with low-level internal echoes in continuity with the affected peripheral nerve. ${ }^{39}$ Internal flow on color and power Doppler imaging is typically present, which is an important finding to differentiate a peripheral nerve sheath tumor from a complex ganglion cyst, as a peripheral nerve sheath tumor may show increased posterior through-transmission similar to a cyst. ${ }^{39}$ A central fibrotic collagenous hyperechoic area and a peripheral hypoechoic myxoid region creating a target

Figure 14. Image from a 59-year-old man with neuroma formation after knee amputation. Sonography image long axis to the common peroneal nerve (arrowheads) shows a hypoechoic neuroma (arrows).

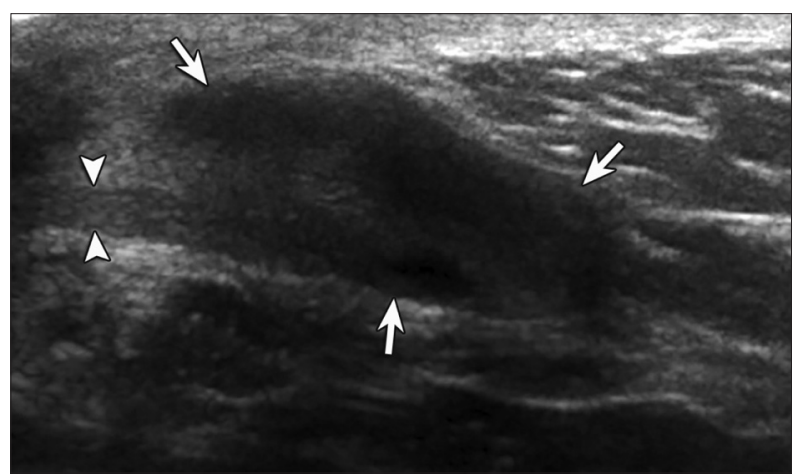

Figure 15. Image from a 59-year old man with an ancient schwannoma. Sonography shows a heterogeneous mass (arrows) with low-level internal echoes and cyst formation in continuity with the ulnar nerve in the arm, representing an ancient schwannoma. Note posterior increased through-transmission (curved arrow).

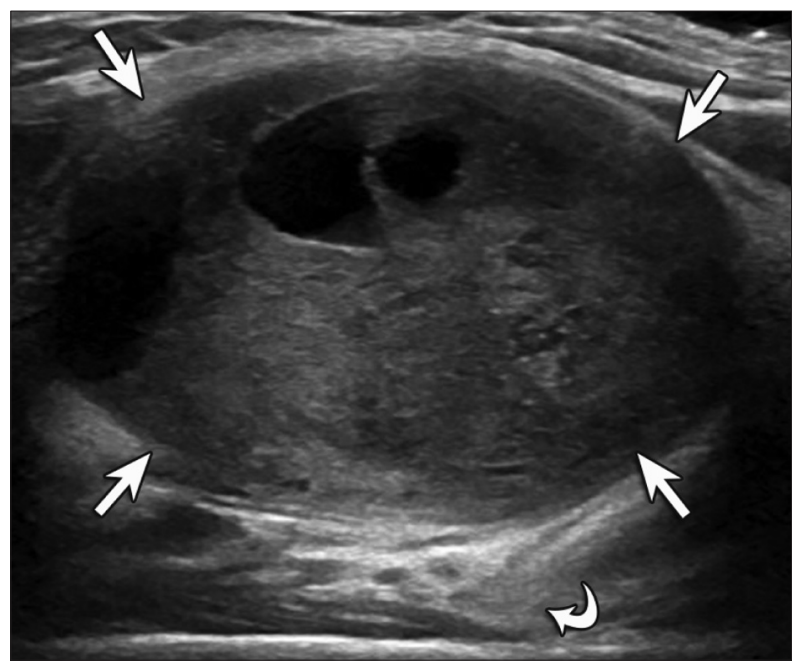


appearance has also been described with neurofibromas. ${ }^{40}$ In addition, neurofibromas tend to be located central and schwannomas eccentric to the affected nerve, although accurate differentiation between a schwannoma and a neurofibroma may be difficult with sonography. ${ }^{41}$ However, the presence of a lobulated contour, a fusiform shape, and hypovascularity suggests a neurofibroma, and the lack of such findings suggests a schwannoma. ${ }^{42}$ While scanning, transducer pressure typically elicits symptoms.

Peripheral nerve sheath tumors may have a variable appearance as well. Large tumors may have cystic regions, whereas an ancient schwannoma may have echogenic and shadowing calcification. A large or enlarging peripheral nerve sheath tumor should raise suspicion for a malignant peripheral nerve sheath tumor; however, sonography is

Figure 16. Image from a 49-year-old man with neurofibroma. Sonography shows a predominantly hypoechoic mass (arrows) with low-level internal echoes in the lower leg, representing a neurofibroma. Note internal flow on color Doppler imaging and posterior increased throughtransmission (curved arrow).

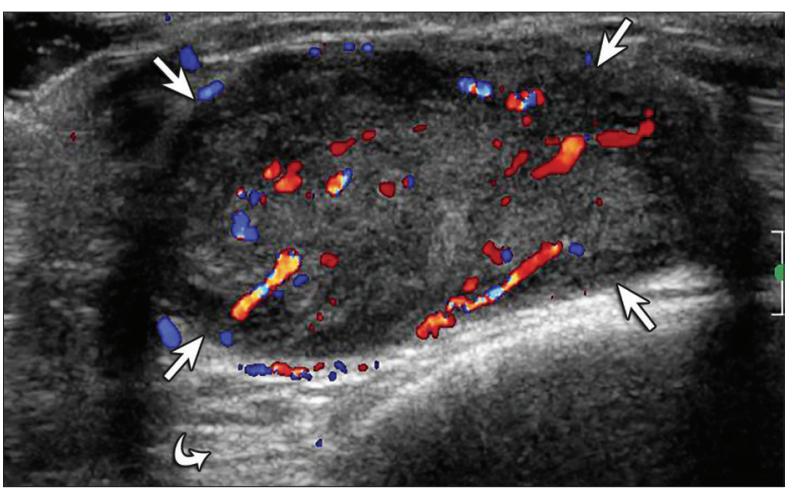

Figure 17. Image from a 51-year-old man with a malignant peripheral nerve sheath tumor in the deltoid muscle. Sonography shows a nonspecific large heterogeneous but predominantly hypoechoic mass (arrows) representing a malignant peripheral nerve sheath tumor.

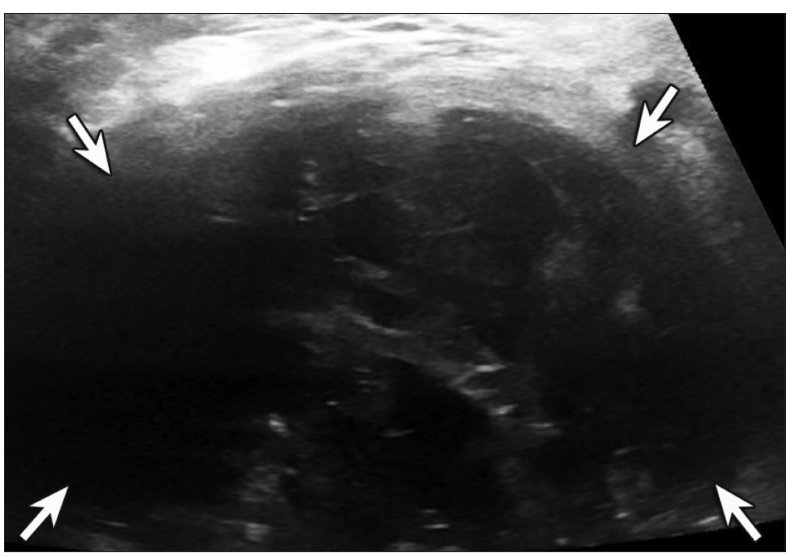

unable to differentiate benign from malignant forms of peripheral nerve sheath tumors (Figure 17).

\section{Summary}

Sonography is an effective method to evaluate peripheral nerves. Entrapment neuropathies will appear as hypoechoic enlargement at the entrapment site. Dynamic assessment is used to evaluate for ulnar nerve dislocation, snapping triceps syndrome, and Morton neuroma. Intraneural ganglion cyst formation can affect the common peroneal nerve related to an articular nerve branch to the proximal tibiofibular joint. Nerve trauma may result in hypoechoic enlargement or complete discontinuity with retraction and hypoechoic neuroma formation. A peripheral nerve sheath tumor appears as a hypoechoic mass with low-level internal echoes in continuity with the affected nerve.

\section{References}

1. Zbojniewicz AM. US for diagnosis of musculoskeletal conditions in the young athlete: emphasis on dynamic assessment. Radiographics 2014; 34:1145-1162.

2. Silvestri E, Martinoli C, Derchi LE, Bertolotto M, Chiaramondia M, Rosenberg I. Echotexture of peripheral nerves: correlation between US and histologic findings and criteria to differentiate tendons. Radiology 1995; 197:291-296.

3. Jacobson JA, Fessell DP, Lobo Lda G, Yang LJ. Entrapment neuropathies I: upper limb (carpal tunnel excluded). Semin Musculoskelet Radiol 2010; 14:473-486.

4. Beekman R, Van Der Plas JP, Uitdehaag BM, Schellens RL, Visser LH Clinical, electrodiagnostic, and sonographic studies in ulnar neuropathy at the elbow. Muscle Nerve 2004; 30:202-208.

5. Beekman R, Schoemaker MC, Van Der Plas JP, et al. Diagnostic value of high-resolution sonography in ulnar neuropathy at the elbow. Neurology 2004; 62:767-773.

6. Klauser AS, Faschingbauer R, Bauer T, et al. Entrapment neuropathies II carpal tunnel syndrome. Semin Musculoskelet Radiol 2010; 14:487-500.

7. Keith MW, Masear V, Amadio PC, et al. Treatment of carpal tunnel syndrome. J Am Acad Orthop Surg 2009; 17:397-405.

8. Peetrons PA, Derbali W. Carpal tunnel syndrome. Semin Musculoskelet Radiol 2013; 17:28-33.

9. Ooi CC, Wong SK, Tan AB, et al. Diagnostic criteria of carpal tunnel syndrome using high-resolution ultrasonography: correlation with nerve conduction studies. Skeletal Radiol 2014; 43:1387-1394.

10. Klauser AS, Halpern EJ, DeZordo T, et al. Carpal tunnel syndrome assessment with US: value of additional cross-sectional area measurements of the median nerve in patients versus healthy volunteers. Radiology 2009; 250:171-177. 
11. Mallouhi A, Pultzl P, Trieb T, Piza H, Bodner G. Predictors of carpal tunnel syndrome: accuracy of gray-scale and color Doppler sonography. AJR Am J Roentgenol 2006; 186:1240-1245.

12. Propeck T, Quinn TJ, Jacobson JA, Paulino AF, Habra G, Darian VB. Sonography and MR imaging of bifid median nerve with anatomic and histologic correlation. AJR Am J Roentgenol 2000; 175:1721-1725.

13. Klauser AS, Halpern EJ, Faschingbauer R, et al. Bifid median nerve in carpal tunnel syndrome: assessment with US cross-sectional area measurement. Radiology 2011; 259:808-815.

14. Lee CH,Kim TK, Yoon ES, Dhong ES. Postoperative morphologic analysis of carpal tunnel syndrome using high-resolution ultrasonography. Ann Plast Surg 2005; 54:143-146.

15. Abicalaf CA, de Barros N, Sernik RA, et al. Ultrasound evaluation of patients with carpal tunnel syndrome before and after endoscopic release of the transverse carpal ligament. Clin Radiol 2007; 62:891-896.

16. Martinoli C, Bianchi S, Gandolfo N, Valle M, Simonetti S, Derchi LE. US of nerve entrapments in osteofibrous tunnels of the upper and lower limbs. Radiographics 2000; 20(special issue):S199-S213.

17. Beekman R, Visser LH, Verhagen WI. Ultrasonography in ulnar neuropathy at the elbow: a critical review. Muscle Nerve 201 1; 43:627-635.

18. Jacobson JA, Jebson PJ, Jeffers AW, Fessell DP, Hayes CW. Ulnar nerve dislocation and snapping triceps syndrome: diagnosis with dynamic sonography_report of three cases. Radiology 2001; 220:601-605.

19. Beltran LS, Bencardino J, Ghazikhanian V, Beltran J. Entrapment neuropathies III: lower limb. Semin Musculoskelet Radiol 2010; 14:501-511.

20. Jain S, Mannan K. The diagnosis and management of Morton's neuroma: a literature review. Foot Ankle Spec 2013; 6:307-317.

21. Cloke DJ, Greiss ME. The digital nerve stretch test: a sensitive indicator of Morton's neuroma and neuritis. Foot Ankle Surg 2006; 12:201-203.

22. Quinn TJ, Jacobson JA, Craig JG, van Holsbeeck MT. Sonography of Morton's neuromas. AJR Am J Roentgenol 2000; 174:1723-1728.

23. Torriani M, Kattapuram SV. Technical innovation. Dynamic sonography of the forefoot: the sonographic Mulder sign. AJR Am J Roentgenol 2003; 180:1121-1123.

24. Lee MJ, Kim S, Huh YM, et al. Morton neuroma: evaluated with ultrasonography and MR imaging. Korean J Radiol 2007; 8:148-155.

25. Spinner RJ, Amrami KK, Rock MG. The use of MR arthrography to document an occult joint communication in a recurrent peroneal intraneural ganglion. Skeletal Radiol 2006; 35:172-179.

26. Spinner RJ, Carmichael SW, Atkinson JL. Intraneural ganglion cyst. J Neurosurg 2006; 104:990-992.

27. Spinner RJ, Desy NM, Amrami KK. Sequential tibial and peroneal intraneural ganglia arising from the superior tibiofibular joint. Skeletal Radiol 2008; 37:79-84.

28. Spinner RJ, Luthra G, Desy NM, Anderson ML, Amrami KK. The clock face guide to peroneal intraneural ganglia: critical "times" and sites for accurate diagnosis. Skeletal Radiol 2008; 37:1091-1099.

29. Spinner RJ, Mokhtarzadeh A, Schiefer TK, Krishnan KG, Kliot M, Amrami KK. The clinico-anatomic explanation for tibial intraneural ganglion cysts arising from the superior tibiofibular joint. Skeletal Radiol 2007; 36:281-292.
30. Spinner RJ, Desy NM, Rock MG, Amrami KK. Peroneal intraneural ganglia, part I: techniques for successful diagnosis and treatment. Neurosurg Focus 2007; 22:E16.

31. Visser LH. High-resolution sonography of the common peroneal nerve: detection of intraneural ganglia. Neurology 2006; 67:1473-1475.

32. Young NP, Sorenson EJ, Spinner RJ, Daube JR. Clinical and electrodiagnostic correlates of peroneal intraneural ganglia. Neurology 2009; $72: 447-$ 452.

33. Grant TH, Omar IM, Dumanian GA, Pomeranz CB, Lewis VA. Sonographic evaluation of common peroneal neuropathy in patients with foot drop. JUltrasound Med 2015; 34:705-711.

34. Tagliafico A, Altafini L, Garello I, Marchetti A, Gennaro S, Martinoli C. Traumatic neuropathies: spectrum of imaging findings and postoperative assessment. Semin Musculoskelet Radiol 2010; 14:512-522.

35. Powell HC, Myers RR. Pathology of experimental nerve compression. Lab Invest 1986; 55:91-100.

36. Zhu J, Liu F, Li D, Shao J, Hu B. Preliminary study of the types of traumatic peripheral nerve injuries by ultrasound. Eur Radio 2011;21:10971101.

37. Umans H, Kessler J, de la Lama M, Magge K, Liebling R, Negron J. Sonographic assessment of volar digital nerve injury in the context of penetrating trauma. AJR Am J Roentgenol 2010; 194:1310-1313.

38. Abreu E, Aubert S, Wavreille G, Gheno R, Canella C, Cotten A. Peripheral tumor and tumor-like neurogenic lesions. Eur J Radiol 2013; 82:38-50.

39. Reynolds DL Jr, Jacobson JA, Inampudi P, Jamadar DA, Ebrahim FS, Hayes CW. Sonographic characteristics of peripheral nerve sheath tumors. AJR Am J Roentgenol 2004; 182:741-744.

40. Lin J,Jacobson JA, Hayes CW. Sonographic target sign in neurofibromas. JUltrasound Med 1999; 18:513-517.

41. Tsai WC, Chiou HJ, Chou YH, Wang HK, Chiou SY, Chang CY. Differentiation between schwannomas and neurofibromas in the extremities and superficial body: the role of high-resolution and color Doppler ultrasonography. JUltrasound Med 2008; 27:161-166.

42. Ryu JA, Lee SH, Cha EY, Kim TY, Kim SM, Shin MJ. Sonographic differentiation between schwannomas and neurofibromas in the musculoskeletal system. J Ultrasound Med 2015; 34:2253-2260. 